\section{Der Nutzen von KRAS bei NSCLC}

Taugt das Signalprotein KRAS wirklich zur Vorhersage, ob ein Patient mit fortgeschrittenem nichtkleinzelligem Lungenkarzinom (NSCLC) auf eine Therapie mit Tyrosinkinaseinhibitoren (TKI) anspricht? Zuletzt waren Zweifel daran aufgekommen. Eine Metaanalyse soll Klarheit schaffen.

Cit einigen Jahren gehören TKI zur Behandlung des NSCLC, denn sie hemmen die Signaltransduktion von Wachstumsfaktorrezeptoren, etwa von EGFR („epidermal growth factor receptor"). Ob Patienten auf TKI ansprechen, lässt sich bislang nur schwer vorhersagen. Viele Kliniken nutzen als Prädiktor den Status des Signalproteins KRAS, dessen kodierendes Gen oft mutiert ist - bei 20 $30 \%$ aller Adeno-, aber nur selten bei Plattenepithelkarzinomen. Eine Rolle scheinen auch Mutationen beim EGFR selbst zu spielen. Doch zuletzt waren Zweifel am prädiktiven Wert von KRAS aufgekommen. Ein Team um den chinesischen Forscher Min Ying ging dem mit einer Metaanalyse nach. Es fand 12 prospektive Interventionsstudien mit insgesamt 1.859 unselektierten Patienten, bei denen es die Rolle einer KRAS-Mutation im Rahmen einer EGFR-TKI-Behandlung untersuchte. Endpunkte waren Gesamt- und progressionsfreies Überleben sowie Gesamtansprechrate.

Der Analyse zufolge war eine KRASMutation mit kürzerem Gesamt- (Hazard Ratio [HR] 2,09) und progressionsfreiem Überleben (HR 1,82) sowie einer niedrigeren Gesamtansprechrate assoziiert (Relatives Risiko 0,25). In einer Subgruppenanalyse wurde der Zusammenhang besser, wenn der EGFR-TKI in zweiter oder höherer Linie gegeben wur- de. Dann betrug die HR für Gesamt- und progressionsfreies Überleben 2,45 bzw. 1,86, beim Ansprechen war der Unterschied nicht mehr signifikant ( $\mathrm{p}=0,153$ ).

Zudem wurden 4 retrospektive Studien analysiert, um die Rolle des KRASStatus bei Patienten mit EGFR-Wildtyp zu beurteilen. Hier war kein Einfluss einer KRAS-Mutation mehr zu erkennen.

Fazit: Bei unselektierten Patienten mit fortgeschrittenem NSCLC könnte das Vorliegen einer KRAS-Mutation ein potenzieller negativer Prädiktor für den Nutzen einer EGFR-TKI-Behandlung sein. Wird der EGFR-Status berücksichtigt, ist ein KRAS-Test aber nur von limitiertem Wert, um Patienten zu identifizieren, die von einer EGFR-TKI-Therapie profitieren werden. Christina Berndt

Ying $M$ et al. Should KRAS mutation still be used as a routine predictor of response to EGFR-TKIs in advanced non-small-cell lung cancer? A revaluation based on meta-analysis. J Cancer Res Clin Oncol. 2015;141(8):1427-39.

\title{
Überlebensvorhersage beim NSCLC
}

Patienten mit nichtkleinzelligem Lungenkarzinom (NSCLC) im Stadium III sind eine heterogene Gruppe. Niederländische Forscher suchten nach einem mathematischen Modell, um ihr Überleben besser vorhersagen zu können.

$\mathrm{N}$ ach dem TN-Staging sind NSCLCPatienten im Stadium III eine homogene Gruppe. Dass das nicht korrekt ist, zeigt sich schon an der großen Divergenz beim Gesamtüberleben. Mittlerweile sind immer mehr Informationen über individuelle Patienten verfügbar (von intensiverer Bildgebung bis hin zu Genomic- und Proteomic-Daten), und zugleich wächst die Zahl der Behandlungsoptionen stetig. So sind zwar individuellere Therapien möglich, aber die Entscheidung darüber wird nicht leichter.

Die Gruppe um Cary Oberije analysierte daher Daten von 548 Patienten mit einem NSCLC im Stadium III und entwickelte auf dieser Basis ein Entscheidungshilfesystem. Das DDS (Decision Support Systems) sollte mit individuellen, prognostisch relevanten Daten gefüttert werden und so eine Voraussage

erlauben, mit welcher Therapie ein Patient die besten Überlebenschancen hat.

Alle Patienten waren entweder bestrahlt worden oder hatten sich einer kombinierten Chemoradiotherapie unterzogen. Im Rahmen einer stratifizierten Cox-Regressionsanalyse wurde mithilfe einer Bootstrap-Prozedur ein Algorithmus erstellt. Das Bootstrapping ist eine statistische Methode des Resampling, bei der auf der Grundlage derselben Stichprobe immer wieder neue Verteilungsfunktionen entwickelt und überprüft werden. Die Zuverlässigkeit des so errechneten Modells wurde sowohl intern als auch mithilfe zweier externer Datensätze $(n=174$ und $n=130)$ validiert. Beim Erstellen des Modells wurden die Art der Therapie, das Geschlecht, der WHO-Performancestatus, die Gesamtbehandlungszeit, die äquivalente Bestrahlungsdosis, die Zahl der positiven
Lymphknoten sowie das Tumorvolumen einbezogen. Berücksichtigt wurde auch das Alter, obwohl es sich als statistisch nicht signifikant erwies. So wurden Nomogramme entwickelt, mit deren Hilfe Voraussagen zum Überleben individueller Patienten gemacht wurden. Letztlich konnte das Modell in den externen Datensammlungen die Risikogruppen identifizieren. Der Test ist auch online verfügbar (https://www.cancerdata.org/ 10.1016/j.ijrobp.2015.02.048).

Fazit: Die Aussagekraft des hier entwickelten Tests ist mit einem c-Wert von 0,62 nur moderat. Allerdings ist sie deutlich höher als die Voraussagen, die sich auf der Grundlage der TNM-Klassifikation machen lassen: Sie erreichen nur Werte von 0,50 bis 0,55 . Das Modell ist also ein lohnender erster Ansatz zur Entwicklung eines verlässlichen Entscheidungshilfesystems beim NSCLC im Stadium III.

Christina Berndt

Oberije C et al. A Validated Prediction Model for Overall Survival From Stage III Non-Small Cell Lung Cancer: Toward Survival Prediction for Individual Patients. Int J Radiat Oncol Biol Phys. 2015:92(4):935-44. 